\title{
Electron-phonon scattering in topological insulator thin films
}

\author{
Sébastien Giraud, Arijit Kundu, and Reinhold Egger \\ Institut für Theoretische Physik, Heinrich-Heine-Universität, D-40225 Düsseldorf, Germany
}

(Dated: May 25, 2022)

\begin{abstract}
We present a theoretical study of electron-phonon scattering effects in thin films made of a strong topological insulator. Phonons are modelled by isotropic elastic continuum theory with stress-free boundary conditions, and the interaction with the helical surface Dirac fermions is mediated by the deformation potential. We determine the temperature-dependent electrical resistivity $\rho(T)$ and the quasiparticle decay rate $\Gamma(T)$ observable in photoemission. The low- and high-temperature power laws for both quantities are obtained analytically. Detailed estimates covering the full temperature range are provided for $\mathrm{Bi}_{2} \mathrm{Se}_{3}$.

PACS numbers: 73.50.Bk, 72.10.Di, 63.22.Dc
\end{abstract}

\section{INTRODUCTION}

The recently discovered state of matter called "topological insulator" (TI) currently represents one of the most active areas in condensed matter physics $\stackrel{1,2}{=}$ TIs are characterized by an insulating gap in the bulk but at the same time have an odd number of gapless surface modes protected against all time-reversal invariant (and sufficiently weak) perturbations $\underline{\underline{3.4}}$ In a three-dimensional (3D) TI, these surface modes correspond to massless twodimensional (2D) Dirac fermions, where the spin direction is in the surface plane and perpendicular to momentum ("spin-momentum locking"). A typical reference material is $\mathrm{Bi}_{2} \mathrm{Se}_{3}$ with a bulk gap $\Delta_{b} \approx 0.3 \mathrm{eV}$. The helical Dirac electron property of the TI surface state has been experimentally confirmed by spin- and angleresolved photoemission spectroscopy (ARPES) 1.5 Transport experiments are more difficult in that respect since the surface contribution is often masked by the residual conductivity due to impurities or defects in the bulk $\underline{\underline{6}-8}$ In thin films made of TI materials, however, the bulk contribution is largely suppressed relative to the surface contribution, rendering the latter easier to observe.

In this paper, we provide a detailed theoretical analysis of both the temperature-dependent resistivity $\rho(T)$ and the quasiparticle lifetime $\Gamma(T)$ (observable in ARPES ${ }^{9.10}$ ) for a thin TI film. The approach taken here generalizes previous work for the semi-infinite geometry (with only one surface) by two of us 11 to the film geometry. This brings about several important changes compared to Ref. 11 that are discussed below. We model the electronic part by retaining only the Dirac surface states obtained from the low-energy bandstructure, $\frac{12}{2}$ and our theory always assumes that the Fermi level is located inside the bulk gap. We note in passing that Ref. 12 provides more accurate parameter estimates as the earlier

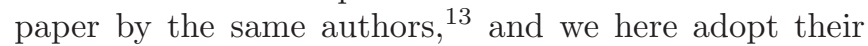
new parameters in our calculations using $\mathrm{Bi}_{2} \mathrm{Se}_{3}$ as example. A similar parameter set has been published in Ref. 14 .

In sufficiently thin films, the hybridization of the two surface states eventually causes insulating behavior, as has recently been observed experimentally from ARPES for $\mathrm{Bi}_{2} \mathrm{Se}_{3}$ films $\frac{15,16}{}$ For $\mathrm{Bi}_{2} \mathrm{Se}_{3}$, several calculations predicted $14,17,18$ a gap $\Delta(L)$ with (as a function of the width $L$ ) oscillations superimposed on an exponential decay. Similar calculations, however, found no oscillations, with a well-established TI phase already for $L \geq 3$ quintuple layers (QLs) $\stackrel{19,20}{=}$ Using the parameter set of Ref. 12, we also find no evidence for oscillations in $\Delta(L)$, see Sec. II below. For large width, one then has (upper and lower) massless Dirac fermion surfaces 21

Our working assumption below is that electron-phonon scattering is the dominant source of quasiparticle decay and backscattering. Electron-electron interactions are indeed expected to give only subleading corrections to the resistivity as long as $T \gtrsim 1 \mathrm{mK}{ }^{22}$ Disorder effects are more likely to compete with phonon-induced backscattering effects. However, for elevated temperatures, $T \gtrsim 100 \mathrm{~K}$, phonon effects dominate even for present-day samples, and anticipating higher purity films in the future, this crossover temperature may be lowered significantly. ARPES setups allowing to test our predictions for the quasiparticle decay rate are basically available,$\frac{5,23-25}{2}$ Other surface scattering techniques have also been applied to extract the phonon dispersions $\stackrel{26}{=} \mathrm{We}$ here follow Ref. 11 and model the phonons using elastic continuum theory. ${ }^{27}$ Since even at room temperature, one effectively probes low energy scales, we keep only longwavelength acoustic phonon modes. For these, previous work on related materials has shown $\underline{28,29}$ that isotropic elastic continuum theory provides a reasonable approximation. The phonon eigenmodes in the thin film geometry and their coupling to electronic modes have previously been determined in the context of semiconductor quantum well structures $\underline{\underline{30}}$ (Note that the semi-infinite case has been treated in Ref. 31.) We basically reproduce the phonon eigenmodes of Ref. 30 , but the coupling to the helical electronic eigenstates in a TI film is different from the semiconductor case. Note that piezoelectric couplings are suppressed by symmetry here,$\stackrel{29}{,}$ and spinphonon type couplings 32 are also expected to be subdominant to the deformation potential taken into account below.

Most TI experiments have so far addressed only optical phonons $\stackrel{33}{=} \mathrm{cf}$. also the corresponding situation 
for Bi surfaces,$\underline{34}$ but very recently ARPES studies reported phonon-induced broadening of the lineshape in TIs ${ }^{23-25}$ The observed $\mathrm{Bi}_{2} \mathrm{Se}_{3}$ electron-phonon coupling strength,$\underline{24}$ which has been extracted from the prefactor in the high-temperature quasiparticle decay rate $\Gamma \propto T$, is in good agreement with our theoretical estimates 11 This indicates that the low-energy approach indeed provides a reasonable starting point. To the best of our knowledge, no detailed measurements for the temperature dependence of the TI film resistivity have been reported so far. We mention in passing that for the related case of a 2D graphene monolayer, a similar comparison of theory $\stackrel{35,36}{\underline{3}}$ to experiment $\frac{37}{\underline{3}}$ has turned out to be successful. Remarkably, the electron-phonon coupling observed in Ref. 24 and independently estimated by us 11 turns out to be quite large. Under room temperature conditions, the resulting lifetime of helical quasiparticles is therefore short, and the resistivity is rather large. This behavior is substantially different from what is found in graphene. We suspect that this is (partially) due to the different Debye temperatures in both materials.

The structure of the remainder of this paper is as follows. In Sec. II we discuss the model for the surface states in the thin film and their coupling to the quantized phonon modes. We then turn to the calculation of the electrical resistivity in Sec. III, followed by the lifetime broadening in Sec. IV] The paper concludes with a brief discussion in Sec. V. Technical details of our calculations can be found in various appendices. Note that we use units with $\hbar=1$.

\section{MODEL}

In this section we describe the model employed in our study of electron-phonon scattering in a TI film. The model parameters below are chosen for $\mathrm{Bi}_{2} \mathrm{Se}_{3}$ as a concrete example. The film has infinite extension in the $x y$ plane and the width $L$, where $|z|<L / 2$. We start by reviewing the construction of the effective surface Hamiltonian describing the (upper and lower) electronic surface states of a TI film.

\section{A. Electronic surface states}

Keeping all terms up to second order in the momentum around the $\Gamma$ point, $\left(k_{x}, k_{y}, k_{z}\right)$, the low-energy physics of 3D TI materials like $\mathrm{Bi}_{2} \mathrm{Se}_{3}$ or $\mathrm{Bi}_{2} \mathrm{Te}_{3}$ is well described by an effective four-band model $\underline{\underline{1}}$ Using the basis states $\left\{\left|P 1_{z}^{+}, \uparrow\right\rangle,\left|P 2_{z}^{-}, \uparrow\right\rangle,\left|P 1_{z}^{+}, \downarrow\right\rangle,\left|P 2_{z}^{-}, \downarrow\right\rangle\right\}$, the lowenergy bulk Hamiltonian reads $12-14$

$$
H=\left(\begin{array}{cccc}
\epsilon_{0}+M & -i A_{1} k_{z} & 0 & A_{2} k_{-} \\
i A_{1} k_{z} & \epsilon_{0}-M & A_{2} k_{-} & 0 \\
0 & A_{2} k_{+} & \epsilon_{0}+M & -i A_{1} k_{z} \\
A_{2} k_{+} & 0 & i A_{1} k_{z} & \epsilon_{0}-M
\end{array}\right)
$$

with $\epsilon_{0}=C+D_{1} k_{z}^{2}+D_{2}\left(k_{x}^{2}+k_{y}^{2}\right), M=M_{0}-B_{1} k_{z}^{2}-$ $B_{2}\left(k_{x}^{2}+k_{y}^{2}\right)$ and $k_{ \pm}=k_{x} \pm i k_{y}$. The model parameters for $\mathrm{Bi}_{2} \mathrm{Se}_{3}$ have been determined from first principles, $\stackrel{12}{=}$

$$
\begin{aligned}
M_{0} & =0.28 \mathrm{eV}, \quad C=-0.0083 \mathrm{eV}, \\
A_{1} & =2.26 \mathrm{eV} \AA, \quad A_{2}=3.33 \mathrm{eV} \AA, \\
B_{1} & =6.86 \mathrm{eV}^{2}, \quad B_{2}=44.5 \mathrm{eV} \AA^{2}, \\
D_{1} & =5.74 \mathrm{eV} \AA, \quad D_{2}=30.4 \mathrm{eV \AA} \AA^{2} .
\end{aligned}
$$

We may write the Hamiltonian (1) in the form $H=H_{0}+$ $H^{\prime}$, where $H_{0}=\left(\begin{array}{cc}h_{0}\left(k_{z}\right) & 0 \\ 0 & h_{0}\left(k_{z}\right)\end{array}\right)$ is the $2 \times 2$ block matrix obtained for $k_{x}=k_{y}=0$, with

$$
h_{0}\left(k_{z}\right)=\left(\begin{array}{cc}
\epsilon_{0}\left(k_{z}\right)+M_{0}-B_{1} k_{z}^{2} & -i A_{1} k_{z} \\
i A_{1} k_{z} & \epsilon_{0}\left(k_{z}\right)-M_{0}+B_{1} k_{z}^{2}
\end{array}\right) .
$$

Note that eigenstates of $H_{0}$ have conserved spin.

In order to find the surface states in the film geometry, we follow the usual strategy $\underline{\underline{12}} \underline{\underline{14}}$ and first look for general bispinor eigenstates of $h_{0}$,

$$
h_{0}\left(k_{z} \rightarrow-i \partial_{z}\right) \Psi(z)=E_{0} \Psi(z) .
$$

The general solution to Eq. (44) reads $(j= \pm, s= \pm)$

$$
\Psi(z)=\sum_{j s} c_{j s} e^{-s \eta_{j} z}\left(\begin{array}{c}
E_{0}-C+M_{0}+\left(D_{1}+B_{1}\right) \eta_{j}^{2} \\
-s A_{1} \eta_{j}
\end{array}\right)
$$

with arbitrary $c_{j s}$ and the inverse lengthscales

$$
\eta_{ \pm}=\left[\left(-\tilde{B} \pm \sqrt{\tilde{B}^{2}-4 \tilde{A} \tilde{C}}\right) /(2 \tilde{A})\right]^{1 / 2},
$$

where $\tilde{A}=D_{1}^{2}-B_{1}^{2}, \tilde{B}=A_{1}^{2}-2\left[M_{0} B_{1}+D_{1}\left(C-E_{0}\right)\right]$, and $\tilde{C}=\left(E_{0}-C\right)^{2}-M_{0}^{2}$. The Dirichlet boundary conditions defining the film geometry, $\Psi(z= \pm L / 2)=0$, then imply the transcendental equation

$$
\frac{\left[E_{0}-C+M_{0}+\left(D_{1}+B_{1}\right) \eta_{+}^{2}\right] \eta_{-}}{\left[E_{0}-C+M_{0}+\left(D_{1}+B_{1}\right) \eta_{-}^{2}\right] \eta_{+}}=\frac{\tanh \left(\eta_{-} L / 2\right)}{\tanh \left(\eta_{+} L / 2\right)},
$$

or the same condition with $\eta_{+} \leftrightarrow \eta_{-}$on the right hand side. Numerical solution of these equations yields the $\Gamma$ point energies $E_{0}^{( \pm)}$. The corresponding eigenstates $\Psi_{ \pm}(z)$ follow from Eq. (5),

$$
\Psi_{ \pm}(z)=\mathcal{N}_{ \pm}\left(\begin{array}{c}
\left(D_{1}+B_{1}\right) \Lambda_{ \pm} F_{\mp}^{ \pm}(z) \\
A_{1} F_{ \pm}^{ \pm}(z)
\end{array}\right),
$$

where the $\mathcal{N}_{ \pm}$are normalization constants and

$$
\Lambda_{ \pm}=\left[\frac{\eta_{+}^{2}-\eta_{-}^{2}}{\eta_{+} \operatorname{coth}^{ \pm}\left(\eta_{+} L / 2\right)-\eta_{-} \operatorname{coth}^{ \pm}\left(\eta_{-} L / 2\right)}\right]_{E_{0}^{( \pm)}}
$$

with $\operatorname{coth}^{+}(y)=\operatorname{coth}(y)$ and $\operatorname{coth}^{-}(y)=\tanh (y)$. Finally, the $F$ functions are

$$
F_{+}^{ \pm}(z)=\left[\frac{\cosh \left(\eta_{+} z\right)}{\cosh \left(\eta_{+} L / 2\right)}-\frac{\cosh \left(\eta_{-} z\right)}{\cosh \left(\eta_{-} L / 2\right)}\right]_{E_{0}^{( \pm)}},
$$


where $F_{-}^{ \pm}$follows with $\cosh \rightarrow \sinh$. Note that the eigenstates $\Psi_{ \pm}(z)$ describe both spin directions $(\sigma=\uparrow$ and $\sigma=\downarrow)$.

We now project the full Hamiltonian $H$ to the basis spanned by the surface states (6). We define Pauli matrices $\tau_{\alpha=x, y, z}$ switching between the two solutions $\Psi_{\tau= \pm}(z)$, Pauli matrices $\sigma_{\alpha}$ in spin space, and use $\tau_{0}$ and $\sigma_{0}$ as identities. With the energy scales

$$
E_{0}=\frac{E_{0}^{(+)}+E_{0}^{(-)}}{2}, \quad \Delta=E_{0}^{(+)}-E_{0}^{(-)},
$$

the low-energy ("surface") Hamiltonian resulting from this projection reads

$$
H_{\mathrm{eff}}=E_{0} \tau_{0} \sigma_{0}+\frac{\Delta}{2} \tau_{z} \sigma_{0}-A_{2} W \tau_{x}\left(k_{x} \sigma_{x}+k_{y} \sigma_{y}\right)+\mathcal{O}\left(\mathbf{k}^{2}\right),
$$

where $W=\left\langle\Psi_{+} \mid \Psi_{-}\right\rangle$. The parameter $\Delta(L)$ is precisely the surface state gap described in the Introduction. For the parameters (2), $\eta_{-}$is always real. However, $\eta_{+}$is real for large $L$ but purely imaginary for small $L$. In any case, we find that $W$ is always real and positive.

Noting that $H_{\text {eff }}$ commutes with $\tau_{z} \sigma_{z}$, it can readily be diagonalized by the unitary transformation $U(\mathbf{k})=$ $\operatorname{diag}\left(U_{+}, U_{-}\right)$, where $\mathbf{k}=\left(k_{x}, k_{y}\right)$ and the $U_{v= \pm}(\mathbf{k})$ are $2 \times 2$ matrices in spin space, with $v$ denoting the eigenvalue of $\tau_{z} \sigma_{z}$. With $\tan \alpha=2 A_{2} W|\mathbf{k}| / \Delta$ and $\tan \theta=$ $k_{y} / k_{x}$, we find

$$
\begin{aligned}
U_{v=+} & =\left(\begin{array}{cc}
e^{-i \theta / 2} \cos (\alpha / 2) & e^{-i \theta / 2} \sin (\alpha / 2) \\
-e^{i \theta / 2} \sin (\alpha / 2) & e^{i \theta / 2} \cos (\alpha / 2)
\end{array}\right), \\
U_{v=-} & =\left(\begin{array}{cc}
-e^{-i \theta / 2} \sin (\alpha / 2) & e^{-i \theta / 2} \cos (\alpha / 2) \\
e^{i \theta / 2} \cos (\alpha / 2) & e^{i \theta / 2} \sin (\alpha / 2)
\end{array}\right) .
\end{aligned}
$$

Switching to second-quantized notation, the eigenstates of $H_{\text {eff }}$ correspond to helical fermions with annihilation operator

$$
c_{\mathbf{k}, v s}=\sum_{\sigma}\left[U_{v}(\mathbf{k})\right]_{\sigma s}^{*} d_{\mathbf{k}, \tau=v \sigma, \sigma}
$$

where $d_{\mathbf{k}, \tau \sigma}$ annihilates a spin- $\sigma$ electron with in-plane momentum $\mathbf{k}$ in the transversal state $\Psi_{\tau}(z)$. The lowenergy electronic Hamiltonian (including the chemical potential $\mu$ ) then takes the final form

$$
H_{\mathrm{el}}=\sum_{\mathbf{k} ; v, s= \pm} \epsilon_{\mathbf{k}, s} c_{\mathbf{k}, v s}^{\dagger} c_{\mathbf{k}, v s}
$$

where the dispersion relation is

$$
\epsilon_{\mathbf{k}, \pm}=E_{0}-E_{0}^{\infty}-\mu \pm \frac{\Delta}{2} \sqrt{1+\left(2 A_{2} W / \Delta\right)^{2} \mathbf{k}^{2}} .
$$

We here choose the zero of energy by setting $E_{0}^{\infty}=$ $C+D_{1} M_{0} / B_{1}=\lim _{L \rightarrow \infty} E_{0}^{( \pm)}$. For the parameters (2), we find $E_{0}^{\infty} \simeq 0.22 \mathrm{eV}$. Moreover, for $L \rightarrow \infty$, the lengthscales $\eta_{ \pm}^{-1}$ are given by $\eta_{+}^{-1} \simeq 12.3 \AA$ and $\eta_{-}^{-1} \simeq 1.9 \AA$. For $k L \gg 1$, the dispersion relation (12) is linear, with

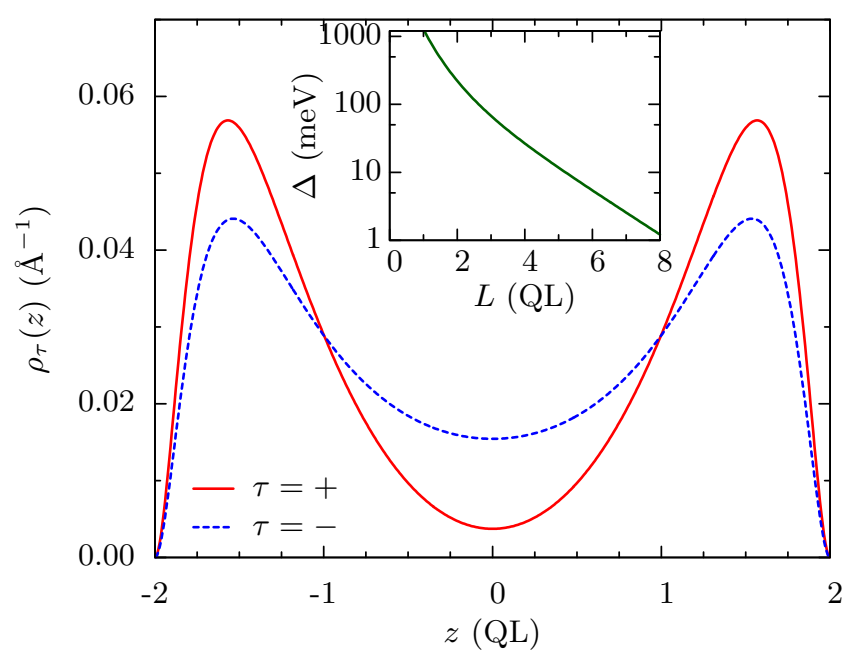

Figure 1: (Color online) Electronic eigenstates for $\mathrm{Bi}_{2} \mathrm{Se}_{3}$ from Eqs. (11) and (2). Main panel: Densities $\rho_{\tau}(z)$ in Eq. (14) for $L=4$ QL. Inset: Gap $\Delta$ vs thickness $L$. Note the semilogarithmic scale.

Fermi velocity $v_{F} \simeq 2.77 \times 10^{5} \mathrm{~m} / \mathrm{s}$. Note that the index $s= \pm$ in Eq. (11) does not correspond to spin anymore.

Similarly, the particle density operator $\hat{n}(\mathbf{r}, z)$ with $\mathbf{r}=$ $(x, y)$ is written in terms of the $d_{\mathbf{k}, \tau \sigma}$ operators,

$$
\hat{n}(\mathbf{r}, z)=\sum_{\mathbf{k}, \mathbf{q}, \tau, \sigma} e^{-i \mathbf{q} \cdot \mathbf{r}} \rho_{\tau}(z) d_{\mathbf{k}+\mathbf{q}, \tau \sigma}^{\dagger} d_{\mathbf{k}, \tau \sigma} .
$$

Using Eq. (10), the density operator (13) can be transformed to the helical basis. We show the single-particle densities for the surface states [Eq. [6)],

$$
\rho_{\tau}(z)=\left[\Psi_{\tau}^{\dagger} \cdot \Psi_{\tau}\right](z)
$$

in Fig. 1 for a film thickness of $L=4 \mathrm{QL}$, where $1 \mathrm{QL} \simeq$ $9.5 \AA$ for $\mathrm{Bi}_{2} \mathrm{Se}_{3}{ }^{38}$ This demonstrates that already for quite thin films, Eq. (6) describes surface states. Note that $\rho_{\tau}(z)$ is an even function of $z$. The inset of Fig. 11 shows the numerically obtained gap $\Delta(L)$, demonstrating the absence of oscillatory behavior for the parameters (21) as well as the exponential decay of $\Delta(L)$ due to the exponentially vanishing overlap of both surface states. We note in passing that for the parameters in Ref. 13, Eq. (7) instead predicts an oscillatory decay of $\Delta(L)$.

\section{B. Phonon model}

We now discuss the long-wavelength acoustic phonon modes in the TI film. We employ isotropic elastic continuum theory, where the longitudinal $\left(c_{l}\right)$ and transverse $\left(c_{t}\right)$ sound velocities correspond to the two Lamé constants $\stackrel{27}{ }$ In $\mathrm{Bi}_{2} \mathrm{Se}_{3}$, they are given ${ }^{39.40}$ by $c_{l} \simeq$ $2900 \mathrm{~m} / \mathrm{s}$ and $c_{t} \simeq 1700 \mathrm{~m} / \mathrm{s}$, respectively. Moreover, the mass density is $\rho_{M}=7680 \mathrm{~kg} / \mathrm{m}^{3} \underline{\underline{41}}$ In order to model the film geometry, we impose stress-free boundary 
conditions 27 at $z= \pm L / 2$. The quantized phonon eigenmodes for this problem have been determined in Ref. 30 . For convenience, we briefly summarize the results next.

Different phonon modes are labeled by a set of quantum numbers, $\Lambda=(\mathbf{q}, \lambda, n)$, where $\mathbf{q}=\left(q_{x}, q_{y}\right)$ is the surface momentum, $\lambda \in(H, S, A)$ denotes the mode type, and $n \in \mathbb{N}$ is a branch index corresponding to the quantization of transverse momentum. The horizontal shear mode $(\lambda=H)$ decouples from all other modes and does not generate a deformation potential, $\underline{\underline{30}}$ and we do not discuss this mode further. We are left with transversally symmetric (dilatational, $\lambda=S$ ) and antisymmetric (flexural, $\lambda=A$ ) phonons. Denoting the dispersion relation of a given phonon mode $\Lambda$ by $\Omega_{\Lambda}$ (see below) and the surface area by $\mathcal{A}$, the displacement field operator is

$$
\mathbf{U}(\mathbf{r}, z, t)=\sum_{\Lambda} \frac{e^{i\left(\mathbf{q} \cdot \mathbf{r}-\Omega_{\Lambda} t\right)}}{\sqrt{2 \rho_{M} \mathcal{A} \Omega_{\Lambda}}} \mathbf{u}_{\Lambda}(z) b_{\Lambda}+\text { H.c. }
$$

where $b_{\Lambda}$ is a bosonic annihilation operator and the noninteracting phonon Hamiltonian is

$$
H_{\mathrm{ph}}=\sum_{\Lambda} \Omega_{\Lambda}\left(b_{\Lambda}^{\dagger} b_{\Lambda}+1 / 2\right) .
$$

The orthonormal eigenmodes $\mathbf{u}_{\Lambda}(z)$ in Eq. (15) describe linear combinations of $e^{ \pm i k_{l, t} z}$ waves, where

$$
k_{l, t}=\sqrt{\left(\Omega_{\Lambda} / c_{l, t}\right)^{2}-q^{2}}
$$

$k_{l, t}=i \kappa_{l, t}$ with $\kappa_{l, t}=\sqrt{q^{2}-\left(\Omega_{\Lambda} / c_{l, t}\right)^{2}}$ for $\Omega_{\Lambda}<c_{l, t} q$. Writing $\mathbf{u}_{\Lambda}(z)$ in the form

$$
\mathbf{u}(z)=\left(i q \phi_{l}-\frac{d \phi_{t}}{d z}\right) \hat{e}_{q}+\left(\frac{d \phi_{l}}{d z}+i q \phi_{t}\right) \hat{e}_{z},
$$

where $\hat{e}_{q}=\mathbf{q} / q$ and

$$
\phi_{l, t}=a_{l, t} \cos \left(k_{l, t} z\right)+b_{l, t} \sin \left(k_{l, t} z\right),
$$

the stress-free boundary conditions at $z= \pm L / 2$ yield

$$
\begin{aligned}
& 2 i q \frac{d \phi_{l}}{d z}-\left(q^{2}-k_{t}^{2}\right) \phi_{t}=0, \\
& 2 i q \frac{d \phi_{t}}{d z}+\left(q^{2}-k_{t}^{2}\right) \phi_{l}=0 .
\end{aligned}
$$

Since both equations have to be fulfilled at $z= \pm L / 2$, we have four linear equations for the four unknown parameters $\left(a_{l, t}, b_{l, t}\right)$. Setting the corresponding determinant to zero, we obtain the following two possibilities. First, for symmetric modes $(\lambda=S)$, we have the condition

$$
\begin{aligned}
\left(q^{2}-k_{t}^{2}\right)^{2} \cos \left(k_{l} L / 2\right) \sin \left(k_{t} L / 2\right) & + \\
4 q^{2} k_{l} k_{t} \sin \left(k_{l} L / 2\right) \cos \left(k_{t} L / 2\right) & =0 .
\end{aligned}
$$

Numerical solution of this transcendental equation obtains the quantized set of dilatational phonon frequencies $\Omega_{\Lambda=(\mathbf{q}, S, n)}$. The corresponding eigenvector, $\mathbf{u}_{\Lambda}(z)$, follows from Eqs. (18) and (19) with $a_{t}=b_{l}=0$ and

$$
a_{l}=\frac{2 \mathcal{N}_{S} q}{\cos \left(k_{l} L / 2\right)}, \quad b_{t}=\frac{i \mathcal{N}_{S}\left(q^{2}-k_{t}^{2}\right)}{k_{t} \cos \left(k_{t} L / 2\right)} .
$$

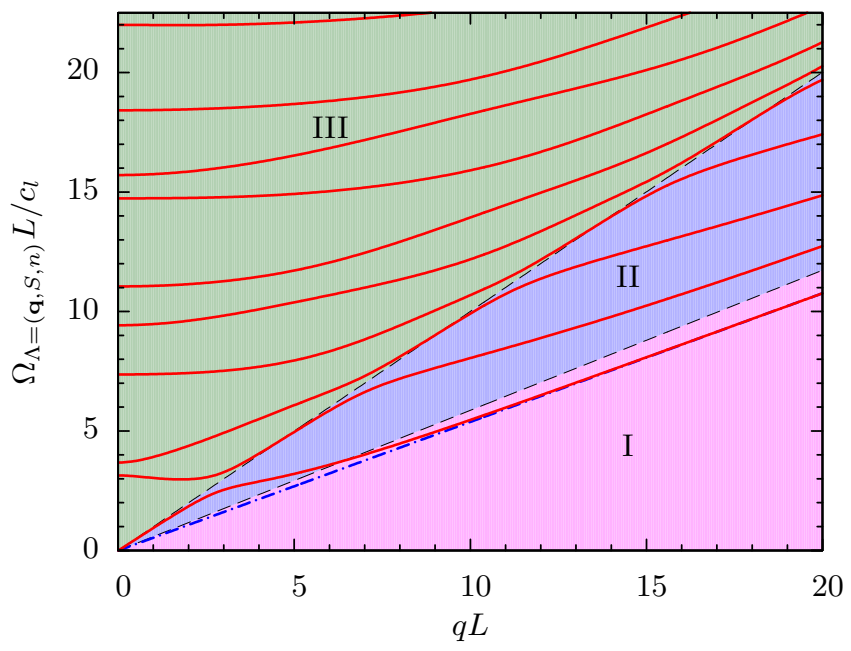

Figure 2: (Color online) Phonon dispersion relation, $\Omega_{\Lambda}$ vs $q$, for the symmetric $(\lambda=S)$ mode (red solid curves). Shown are the ten lowest branches corresponding to the index $n$. Dashed lines separate regions I, II, and III (see main text). The dash-dotted line gives the dispersion relation in Eq. (24); note that the $n=1$ mode coincides with the Rayleigh mode for $q L \gg 1$.

Second, for antisymmetric modes $(\lambda=A)$, we arrive again at the condition in Eq. (21) but with the exchange $\cos \leftrightarrow \sin$. Solving that equation yields the set $\Omega_{\Lambda=(\mathbf{q}, A, n)}$ of quantized flexural phonon modes. The eigenvector $\mathbf{u}_{\Lambda}(z)$ follows again from Eqs. (18) and (19), where now $a_{l}=b_{t}=0$ and

$$
b_{l}=\frac{2 \mathcal{N}_{A} q}{\sin \left(k_{l} L / 2\right)}, \quad a_{t}=\frac{-i \mathcal{N}_{A}\left(q^{2}-k_{t}^{2}\right)}{k_{t} \sin \left(k_{t} L / 2\right)} .
$$

The normalization factors $\mathcal{N}_{\lambda=S, A}$ appearing in Eqs. (22) and (23) are given in Appendix $\mathrm{A}$.

Numerical solution of Eq. (21) yields the spectrum, $\Omega_{\Lambda}$, for the symmetric mode $(\lambda=S)$. The result is shown in Fig. 2. We distinguish three different regions, namely a case where both $k_{l}$ and $k_{t}$ are purely imaginary (region I), when only $k_{l}$ is purely imaginary but $k_{t}$ is real (region II), and finally a case where both $k_{l}$ and $k_{t}$ are real (region III). We observe from Fig. 2 that the $n=1$ mode is the finite-width analogue of the well-known Rayleigh surface mode. 27.31 For the semi-infinite geometry, the Rayleigh mode is the lowest-lying phonon $\stackrel{42}{=}$ It has the dispersion relation

$$
\Omega=c_{R} q, \quad c_{R} \simeq 0.92 c_{t} .
$$

In fact, for $q L \gg 1$, both Eq. (21) and the corresponding equation for $\lambda=A$ reduce to

$$
\left(q^{2}+\kappa_{t}^{2}\right)^{2}=4 q^{2} \kappa_{t} \kappa_{l} .
$$

As discussed in Ref. 27, this equation readily yields the sound velocity $c_{R}$ of the Rayleigh mode. 


\section{Electron-phonon coupling}

The dominant coupling of the above phonon modes to the electronic surface states comes from the deformation potential, $\stackrel{11}{1}$ which couples the local electronic density $\hat{n}(\mathbf{r}, z)[\mathrm{Eq}$. (13)] to the divergence of the displacement vector, $\nabla \cdot \mathbf{U}(\mathbf{r}, z)$, see Eq. (15). Since the surface state density $\rho_{\tau}(z)$ in Eq. (14) is even in $z$, the antisymmetric phonon mode $(\lambda=A)$ does not couple to the surface states. We therefore keep only the symmetric phonon mode from now on (and omit the index $\lambda=S$ ). Transforming Eq. (13) to the helical basis, see Eq. (10), the second-quantized electron-phonon coupling Hamiltonian reads

$$
H_{\mathrm{e}-\mathrm{ph}}=\frac{\alpha}{\sqrt{\mathcal{A}}} \sum_{\mathbf{q}, \mathbf{k}, n ; v, s, s^{\prime}} M_{\mathbf{k}, \mathbf{q}, n}^{\left(v, s, s^{\prime}\right)} b_{\mathbf{q}, n} c_{\mathbf{k}+\mathbf{q}, v s}^{\dagger} c_{\mathbf{k}, v s^{\prime}}+\text { H.c. },
$$

where the $M$ matrix elements involve the unitary matrices $\left[U_{v}(\mathbf{k})\right]_{s \sigma}$ in Eq. (9),

$$
M_{\mathbf{k}, \mathbf{q}, n}^{\left(v, s, s^{\prime}\right)}=-\frac{1}{\sqrt{2 \rho_{M} \Omega_{q, n}}}\left(\frac{\Omega_{q, n}}{c_{l}}\right)^{2} \sum_{\sigma}\left[U_{v}(\mathbf{k}+\mathbf{q})\right]_{s \sigma}^{*}\left[U_{v}(\mathbf{k})\right]_{\sigma s^{\prime}} \int_{-L / 2}^{L / 2} d z \rho_{\tau=v \sigma}(z) \phi_{l}(z)
$$

with the phonon dispersion $\Omega_{q, n}$ in Fig. 2 $\phi_{l}$ is given by Eqs. (19) and (22). The deformation potential strength $\alpha$ in Eq. 25) can be estimated as follows. The hightemperature behavior of the on-shell imaginary part of the electronic self-energy is (see Sec. IV)

$$
\operatorname{Im} \Sigma(k, T)=-\pi \lambda_{k} k_{B} T
$$

which allows to experimentally extract the dimensionless effective electron-phonon coupling constant $\lambda_{k}$. The relation (27) has been observed for $\mathrm{Bi}_{2} \mathrm{Se}_{3}$ in ARPES experiments, $\stackrel{24}{,}$ and $\lambda=0.25 \pm 0.05$ has been measured. In these experiments, the Fermi level was near the bottom of the conduction band, $\mu \simeq 0.28 \mathrm{eV}$, and $k$ in Eq. (27) corresponds to energies $\approx 50$ to $100 \mathrm{meV}$ above the Dirac point. Computing $\lambda_{k}$ within our model, see Sec. IV] the observed value for $\lambda$ corresponds to $\alpha=(30 \pm 8) \mathrm{eV}$. We employ the value $\alpha=30 \mathrm{eV}$ below.

The total Hamiltonian employed in the following sections is then given by $H=H_{\mathrm{el}}+H_{\mathrm{ph}}+H_{\mathrm{e}-\mathrm{ph}}$, see Eqs. (11), (16) and (25). We first address the phononinduced resistivity $\rho$ in Sec. III and then turn to the quasiparticle lifetime in Sec. IV

\section{RESISTIVITY}

Here we discuss the $T$-dependent phonon contribution to the electrical resistivity, $\rho$, in the TI film, using the Hamiltonian described in Sec. II As explained in Sec. IIC, only symmetric (dilatational) phonon modes can cause a finite resistivity for the low-energy surface states within the bulk gap. We compute $\rho$ within the framework of the linearized Boltzmann equation, which has also been employed previously for the related graphene case 35,36 The resulting quasiclassical estimate for $\rho$ is valid 35 as long as $\rho$ is small compared to the resistance quantum, $\rho \ll h / e^{2} \simeq 25.8 \mathrm{k} \Omega$. We sketch the standard derivation $30,35,36,44$ for $\rho$ in Appendix B. The result takes the form

$$
\frac{1}{\rho}=\frac{e^{2}}{2} \sum_{v, s= \pm} \int \frac{d \mathbf{k}}{(2 \pi)^{2}} v_{\mathbf{k}, s}^{2} \tau_{v}\left(\epsilon_{\mathbf{k}, s}\right)\left[-\partial_{\epsilon} n_{F}\left(\epsilon_{\mathbf{k}, s}\right)\right]
$$

where the dispersion relation for helical fermions [Eq. (12)] defines the group velocity, $v_{\mathbf{k}, s}=\hat{e}_{k} \cdot \nabla_{\mathbf{k}} \epsilon_{\mathbf{k}, s}$. Moreover, $n_{F}(\epsilon)$ is the Fermi function, and the energydependent electron-phonon transport scattering rate (inverse time) is

$$
\frac{1}{\tau_{v}\left(\epsilon_{\mathbf{k}, s}\right)}=\sum_{\mathbf{q}, s^{\prime}}\left(1-\frac{v_{\mathbf{k}+\mathbf{q}, s^{\prime}}}{v_{\mathbf{k}, s}} \cos \theta_{\mathbf{k}, \mathbf{q}}\right) \frac{1-n_{F}\left(\epsilon_{\mathbf{k}+\mathbf{q}, s^{\prime}}\right)}{1-n_{F}\left(\epsilon_{\mathbf{k}, s}\right)} W_{(\mathbf{k}, v s) \rightarrow\left(\mathbf{k}+\mathbf{q}, v s^{\prime}\right)}
$$


where $\theta_{\mathbf{k}, \mathbf{q}}$ is the angle between $\mathbf{k}$ and $\mathbf{k}+\mathbf{q}$, and the transition probabilities are obtained from Fermi's golden rule. Using Eq. (25), we find

$$
W_{(\mathbf{k}, v s) \rightarrow\left(\mathbf{k}+\mathbf{q}, v s^{\prime}\right)}=\frac{2 \pi \alpha^{2}}{\mathcal{A}} \sum_{n ; \nu= \pm} \nu n_{B}\left(\nu \Omega_{q, n}\right)\left|M_{\mathbf{k}, \mathbf{q}, n}^{\left(s^{\prime}, s\right)}\right|^{2} \delta\left(\epsilon_{\mathbf{k}, s}+\nu \Omega_{q, n}-\epsilon_{\mathbf{k}+\mathbf{q}, s^{\prime}}\right),
$$

where $n_{B}(\epsilon)$ is the Bose function. While the $M$ matrix elements (26) depend on the index $v= \pm$, we note that $|M|^{2}$ and therefore the transition probabilities $W$ are $v$ independent. This also implies that $\tau_{v}$ does actually not depend on $v$.

With the polar angle $\theta$ between $\mathbf{k}$ and $\mathbf{q}$, such that

$$
\cos \theta_{\mathbf{k}, \mathbf{q}}=\frac{k+q \cos \theta}{\sqrt{k^{2}+q^{2}+2 k q \cos \theta}}
$$

the angular integration in Eq. (29) can be encapsulated in the "transport Eliashberg function", see also Ref. 11,

$$
\mathcal{F}_{k, n, s}^{(\nu)}(q)=\sum_{s^{\prime}} \int_{-\pi}^{\pi} \frac{d \theta}{2 \pi}\left[1-\frac{v_{\mathbf{k}+\mathbf{q}, s^{\prime}}}{v_{\mathbf{k}, s}} \cos \theta_{\mathbf{k}, \mathbf{q}}\right]\left|M_{\mathbf{k}, \mathbf{q}, n}^{\left(s^{\prime}, s\right)}\right|^{2} \delta\left(\epsilon_{\mathbf{k}, s}+\nu \Omega_{q, n}-\epsilon_{\mathbf{k}+\mathbf{q}, s^{\prime}}\right) .
$$

This allows us to write the momentum relaxation rate 29) in the form

$$
\frac{1}{\tau\left(\epsilon_{\mathbf{k}, s}\right)}=\alpha^{2} \sum_{n, \nu} \int_{0}^{\infty} q d q \mathcal{F}_{k, n, s}^{(\nu)}(q) \nu n_{B}\left(\nu \Omega_{q, n}\right) \frac{1-n_{F}\left(\epsilon_{\mathbf{k} s}+\nu \Omega_{q, n}\right)}{1-n_{F}\left(\epsilon_{\mathbf{k} s}\right)} .
$$

The $\theta$-integration in Eq. (32) can then be carried out analytically. We quote the (lengthy) result in Appendix C. which is useful when computing $\mathcal{F}$ numerically. For low temperatures, the quasi-elastic approximation, $\Omega_{q, n} \ll$ $\sqrt{(\Delta / 2)^{2}+\left(A_{2} W k\right)^{2}}$, is applicable and allows to simplify the full result for $\mathcal{F}$ to the $\nu$-independent form

$$
\begin{aligned}
\mathcal{F}_{k, n, s}(q) & =\Theta(2 k-q) \frac{1}{\pi \sqrt{(2 k / q)^{2}-1}} \\
& \times\left.\frac{\sqrt{(\Delta / 2)^{2}+\left(A_{2} W k\right)^{2}}}{\left(A_{2} W k\right)^{2}}\left|M_{\mathbf{k}, \mathbf{q}, n}^{(s, s)}\right|_{\theta_{0}}^{2}\right|^{2}
\end{aligned}
$$

where $\theta=\theta_{0}$ (see Appendix $[$ ) determines the polar angle between $\mathbf{k}$ and $\mathbf{q}$ appearing in the matrix element $M$, and the Heaviside function is denoted by $\Theta(y)$. Note that there is no contribution from interband transitions at low temperatures.

The crossover temperature from the low- to the hightemperature behavior in this system is set ${ }^{11,35}$ by the Bloch-Grüneisen temperature,

$$
T_{\mathrm{BG}}=2 k_{F} c_{R} / k_{B},
$$

with the Rayleigh velocity $c_{R}$ in Eq. (24). $k_{F}(L)$ is defined by $\epsilon_{k_{F}, s=+}=0$ with the dispersion relation (12). For $T \ll T_{\mathrm{BG}}$, the $\mathcal{F}$ function can be approximated by the quasi-elastic expression [Eq. (34)]. It receives the dominant contribution from the $n=1$ branch corresponding to the Rayleigh surface phonon. For small $q$, we find $\Omega_{q, n=1}=c_{s} q$ with $c_{s}=2754 \mathrm{~m} / \mathrm{s}$ (which is slightly below $c_{l}$ ), see also Fig. 2. In addition, we have $\phi_{l}(z)=2\left(c_{t} / c_{s}\right)^{2} /(q \sqrt{L})$ and $c_{s} \ll \min \left(\left|v_{k_{F}}\right|, A_{2} W\right)$, leading to

$$
\mathcal{F}_{k_{F}, 1, \pm}(q)=\frac{\left(c_{t} / c_{l}\right)^{4}}{\pi \rho_{M}\left|v_{k_{F}}\right| c_{s} k_{F}^{2}} \frac{q^{2}}{L}
$$

This allows us to perform all remaining integrations and yields a $T^{4}$ law for the resistivity at low temperatures,

$$
\rho\left(T \ll T_{\mathrm{BG}}\right)=\frac{h}{e^{2}} A\left(\frac{T}{T_{\mathrm{BG}}}\right)^{4},
$$

where the dimensionless prefactor $A$ is

$$
\begin{aligned}
A & =\frac{8 \gamma k_{F} \alpha^{2}}{\pi \rho_{M} v_{k_{F}}^{2} c_{s}}\left(\frac{c_{t} c_{R}}{c_{l} c_{s}}\right)^{4} \frac{1}{L} \\
\gamma & =\left[\int_{-\infty}^{\infty} d x \frac{2 e^{x}}{\left[\left(\pi^{2}+x^{2}\right)\left(e^{x}+1\right)\right]^{2}}\right]^{-1} \simeq 68.4295
\end{aligned}
$$

For $L \rightarrow \infty, A$ obviously vanishes. This suggests that for elevated temperatures (but still $T<T_{\mathrm{BG}}$ ) and finite $L$, the $T^{4}$ law is replaced by the $L$-independent $\rho \propto T^{5}$ law found in Ref. 11. We can estimate the crossover 


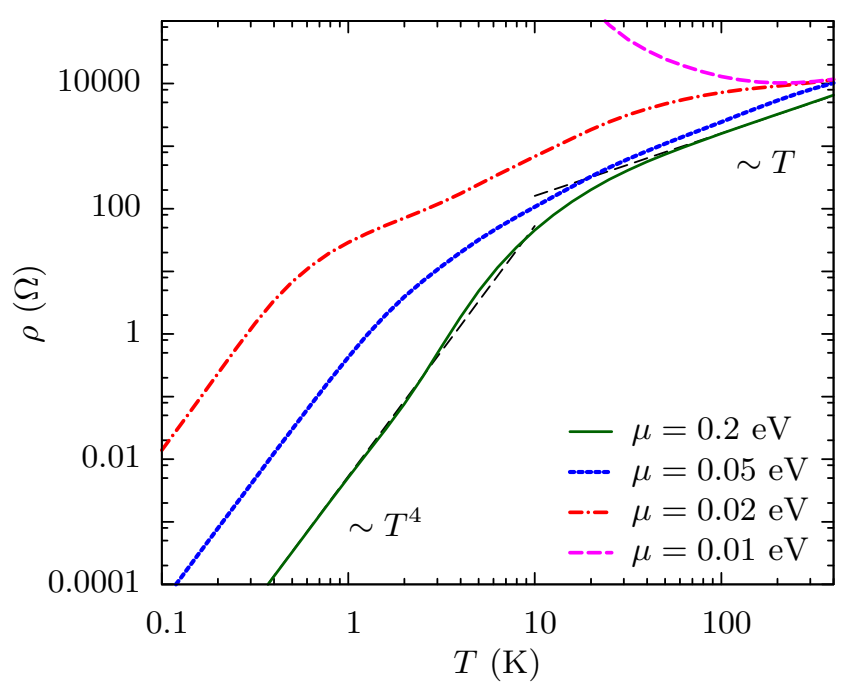

Figure 3: (Color online) Phonon contribution to the resistivity $\rho$ vs temperature $T$ for a TI film of width $L=4 \mathrm{QL}$ and several values of the chemical potential $\mu$. Dashed lines indicate the analytical results for low [Eq. (36)] and high [Eq. (38)] temperatures. Note the double-logarithmic scale.

temperature $T_{c}$ as follows. For $T<T_{\mathrm{BG}}$, we expect an expansion of the form

$$
\left(e^{2} / h\right) \rho=A\left(T / T_{\mathrm{BG}}\right)^{4}+\frac{B}{4}\left(T / T_{\mathrm{BG}}\right)^{5},
$$

with $A \propto 1 / L$ in Eq. (36) and the $L$-independent constant $B$ given in Ref. 11. The crossover from the $T^{4}$ law (for $T \lesssim T_{c}$ ) to the $T^{5}$ law (for $T_{c} \lesssim T<T_{\mathrm{BG}}$ ) thus happens around the temperature $T_{c}=(4 A / B) T_{\mathrm{BG}}$. This gives $T_{c} \simeq 0.14 T_{\mathrm{BG}} /\left(k_{F} L\right)$, which is independent of the chemical potential since $T_{\mathrm{BG}} \propto k_{F}$. For $L=4 \mathrm{QL}$, we obtain $T_{c} \approx 0.9 \mathrm{~K}$. The $T^{4}$ law can thus only be observed for very thin and clean TI films.

In the opposite high-temperature limit, essentially all phonon branches indexed by $n$ contribute to the transport Eliashberg function (32), see Appendix C. Then the relaxation rate $\tau^{-1}\left(\epsilon_{\mathbf{k}, s}\right)$ in Eq. (33) is basically a linear function of the energy. Since the linear term does not contribute to $\rho$ after integration in Eq. (28), we obtain the approximation $1 / \rho \simeq\left(e^{2} / h\right) v_{k_{F}} k_{F} \tau(\epsilon=0)$, where Eq. (33) yields the linear high-temperature law

$$
\rho\left(T \gg T_{\mathrm{BG}}\right)=\frac{h}{e^{2}} C \frac{T}{T_{\mathrm{BG}}}
$$

with the dimensionless prefactor

$$
C=\frac{2 \alpha^{2} c_{R}}{v_{k_{F}}} \sum_{n, \nu= \pm} \int_{0}^{\infty} q d q \frac{\mathcal{F}_{k_{F}, n,+}^{(\nu)}(q)}{\Omega_{q, n}} .
$$

Next we show the full temperature dependence of $\rho$ obtained numerically for a fixed width $L=4 \mathrm{QL}$ and several values of the chemical potential $\mu$, see Fig. 3 ,

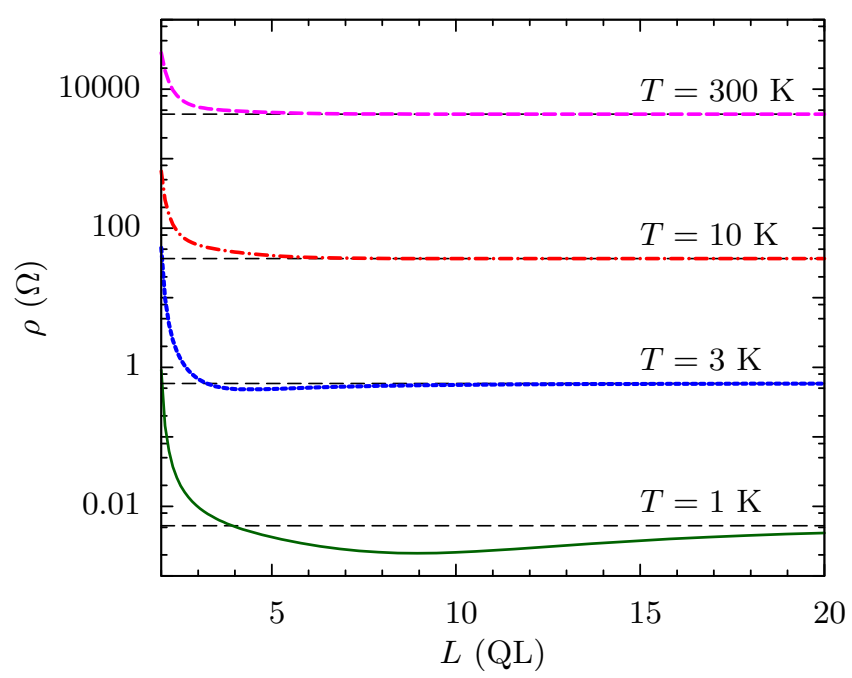

Figure 4: (Color online) Width $(L)$ dependence of the phonon contribution to the resistivity $\rho$ for $\mu=0.2 \mathrm{eV}$ and several temperatures. The dashed horizontal line indicates onequarter of the resistivity $\rho_{\infty}(T)$ in the semi-infinite geometry with otherwise identical parameters $\underline{11}$

In that case, when measured relative to $E_{0}^{\infty}$, we have $E_{0}^{+} \simeq 16 \mathrm{meV}$ and $\Delta / 2 \simeq 13 \mathrm{meV}$. For the lowest $\mu$ in Fig. 3, the Fermi level is thus located inside the surface gap and one has a very large resistivity, where the quasiclassical approach is not reliable in any case. For low temperatures, $T<T_{\mathrm{BG}}$, the analytical result (36) with $\rho \propto T^{4}$ is nicely reproduced by numerics. In this temperature regime, only the Rayleigh mode $(n=1)$ is relevant, similar to what one finds in the semi-infinite geometry 11 In the high-temperature limit, both the $\rho \propto T$ scaling and the prefactor $C$ in Eq. (39) are also consistent with our numerical findings.

Finally, Fig. 4 shows the width $(L)$ dependence of $\rho$ at fixed chemical potential and for several $T$. Two noteworthy observations can be drawn from Fig. 4. First, for low temperatures we observe a "dip" in Fig. 4, where $\rho(L)<\rho(L \rightarrow \infty)$ for intermediate values of $L$. Second, for $L \rightarrow \infty, \rho(L)$ approaches $1 / 4$ of the single-surface value $\rho_{\infty}(T)$ obtained for the semi-infinite geometry $\underline{11}$ Naively, we would expect $\rho(L \rightarrow \infty)=\rho_{\infty} / 2$ because of the presence of two surfaces in the film geometry. This discrepancy indicates that the $L \rightarrow \infty$ limit is singular, and it is not possible to really decouple both surfaces in such an interacting system; see also Ref. 21 for a related discussion.

\section{LIFETIME BROADENING}

Next we discuss the quasiparticle lifetime (inverse decay rate) for the surface fermions in the TI film due to their coupling to phonons, see $H_{\mathrm{e}-\mathrm{ph}}$ in Eq. (25), which implies a finite linewidth of ARPES spectral features. The decay rate, $\Gamma_{k}(T)=-2 \operatorname{Im} \Sigma$, follows from the 


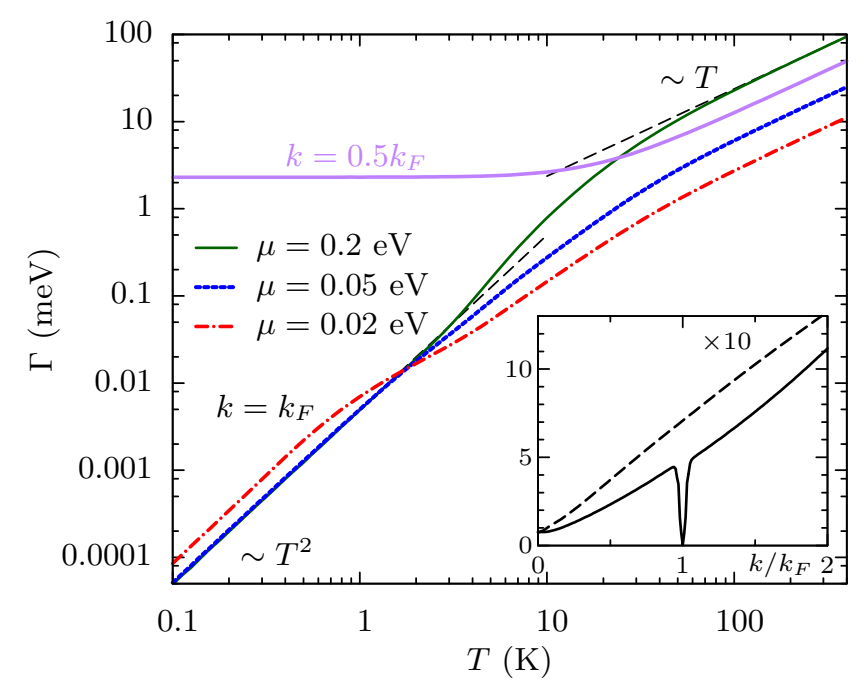

Figure 5: (Color online) Main panel: $T$-dependence of the decay rate $\Gamma$ of a TI film of width $L=4 \mathrm{QL}$ for $k=k_{F}$ and $k=0.5 k_{F}$. For $k=0.5 k_{F}$, only the $\mu=0.2 \mathrm{eV}$ result is displayed. Dashed lines indicate the low- and high-temperature laws $\left(\Gamma \propto T^{2}\right.$ and $\left.\propto T\right)$, respectively. Inset: $k$-dependence of $\Gamma$ for $\mu=0.2 \mathrm{eV}$ and two different temperatures: $T=3 \mathrm{~K}$ (solid line) and $T=300 \mathrm{~K}$ (dashed line; the shown result has to be multiplied by 10).

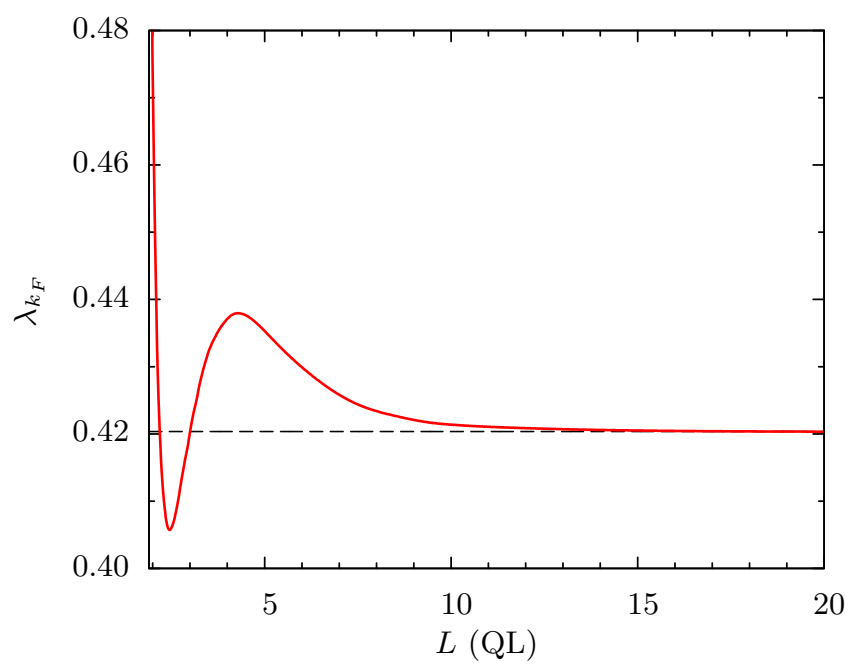

Figure 6: (Color online) Width $(L)$ dependence of the effective electron-phonon coupling constant at the Fermi level $\lambda_{k_{F}}$ for $\mu=0.2 \mathrm{eV}$. The dashed horizontal line indicates one-half of the effective coupling constant in the semi-infinite geometry with otherwise identical parameters. ${ }^{11}$

imaginary part of the on-shell self-energy $\Sigma_{s=+}(\mathbf{k}, \omega=$ $\left.\epsilon_{\mathbf{k}, s=+}\right) \stackrel{45}{=}$ Expanding up to second order in $H_{\mathrm{e}-\mathrm{ph}}$, the "rainbow" diagram yields the self-energy

$$
\begin{aligned}
\Sigma_{s}(\mathbf{k}, \omega) & =\alpha^{2} \sum_{n, s^{\prime}} \int \frac{d \mathbf{q}}{(2 \pi)^{2}}\left|M_{\mathbf{k}, \mathbf{q}, n}^{\left(s^{\prime}, s\right)}\right|^{2} \\
& \times \sum_{\nu= \pm} \nu \frac{n_{B}\left(\nu \Omega_{q, n}\right)+n_{F}\left(\epsilon_{\mathbf{k}+\mathbf{q}, s^{\prime}}\right)}{\omega+i 0^{+}+\nu \Omega_{q, n}-\epsilon_{\mathbf{k}+\mathbf{q}, s^{\prime}}} .
\end{aligned}
$$

Introducing the Eliashberg function $F_{k, n, s}^{(\nu)}(q)$ exactly as the transport Eliashberg function $\mathcal{F}$ in Eq. (32) but without the factor $\left[1-\left(v_{\mathbf{k}+\mathbf{q}, s^{\prime}} / v_{\mathbf{k}, s}\right) \cos \theta_{\mathbf{k}, \mathbf{q}}\right]$, the quasiparticle decay rate follows as

$$
\begin{aligned}
\Gamma_{k}(T) & =\alpha^{2} \sum_{n, \nu} \int_{0}^{\infty} q d q F_{k, n,+}^{(\nu)}(q) \\
& \times\left[n_{B}\left(\Omega_{q, n}\right)+n_{F}\left(\Omega_{q, n}+\nu \epsilon_{k,+}\right)\right] .
\end{aligned}
$$

Expanding this result for high temperatures, $T \gg T_{\mathrm{BG}}$, as in Sec. III] yields, see also Eq. (27), a linear Tdependence,

$$
\begin{aligned}
\Gamma_{k}\left(T \gg T_{\mathrm{BG}}\right) & =2 \pi \lambda_{k} k_{B} T, \\
\lambda_{k} & =\frac{\alpha^{2}}{2 \pi} \sum_{n, \nu} \int_{0}^{\infty} q d q \frac{F_{k, n,+}^{(\nu)}(q)}{\Omega_{q, n}} .
\end{aligned}
$$

The $L$-dependence of $\lambda_{k}$ is shown for $k=k_{F}$ in Fig. 6. We observe an oscillatory dependence, with a saturation at one-half of the corresponding semi-infinite result.

For low temperatures and $k=k_{F}$, the decay rate is dominated by the $n=1$ phonon mode with $q \rightarrow 0$. After some algebra, we find that this implies a $T^{2}$ law,

$$
\Gamma_{k_{F}}\left(T \ll T_{\mathrm{BG}}\right)=\frac{4 \pi\left(c_{t} / c_{l}\right)^{4}\left(k_{F} c_{R} \alpha\right)^{2}}{\rho_{M}\left|v_{k_{F}}\right| c_{s}^{3}} \frac{1}{L}\left(\frac{T}{T_{\mathrm{BG}}}\right)^{2} .
$$

Again, when $T \gtrsim T_{c}$, the $T^{2}$ law (which scales $\propto$ $1 / L$ ) competes with the $L$-independent $T^{3}$ law found in Ref. 11, see Sec. III]. Finally, when $k \neq k_{F}$ and $T \ll T_{\mathrm{BG}}$, the quasiparticle decay rate saturates at the finite value

$$
\Gamma_{k \neq k_{F}}=\alpha^{2} \sum_{n} \int_{0}^{\infty} q d q \Theta\left(\left|\epsilon_{k+}\right|-\Omega_{q, n}\right) F_{k, n,+}^{(\nu)}(q) .
$$

with $\nu=\operatorname{sgn}\left(k_{F}-k\right)$.

Figure 6 shows that the $L \rightarrow \infty$ limit of the decay rate always tends to $\Gamma_{\infty}(T) / 2$, where $\Gamma_{\infty}$ is the corresponding decay rate for the semi-infinite geometry $\underline{\underline{11}}$ This discrepancy with the naive expectation $\Gamma(L \rightarrow \infty)=\Gamma_{\infty}$ has the same origin as the anomalous factor $1 / 2$ appearing in the large- $L$ behavior of the resistivity discussed in Sec. III.

\section{CONCLUSIONS}

In this paper we have studied the effects of longwavelength acoustic phonons on the topologically protected surface fermions in topological insulator films. 
Our model employs the established low-energy electronic Hamiltonian and an isotropic elastic continuum approach for the phonons, with the deformation coupling providing the dominant interaction mechanism. The electronphonon coupling turns out to be surprisingly strong, in accordance with recent ARPES results $\underline{24}$

Using a quasiclassical approach, we have computed the temperature-dependent resistivity of the film due to phonon backscattering, and found a linear $T$ dependence above the Bloch-Grüneisen temperature. In this temperature regime, the phonon-induced resistivity can overcome the disorder-induced ( $T$-independent) contribution and should be observable with present samples. Similarly, the linear $T$ dependence of the quasiparticle decay rate found here is observable 24 in ARPES experiments. The low-temperature behaviors of the resistivity and of the quasiparticle decay rate are probably more difficult to observe.

An interesting extension of our work would be to include the effects of a magnetic field. Magnetotransport measurements in thin films were recently performed 46 and found clear evidence for Landau level formation associated with the massless Dirac fermions forming on both surfaces. The observed broadening of the Landau levels was assigned to disorder and/or interaction effects, but at elevated temperatures, our analysis indicates that electron-phonon interactions may be relevant as well.

\section{Acknowledgments}

We thank Philip Hofmann for useful discussions. This work was supported by the Humboldt foundation and by the SFB TR 12 of the DFG.

\section{Appendix A: Normalization constants}

Here we provide the normalization constants $\mathcal{N}_{S, A}$ appearing in Eqs. (22) and (23) in Sec. IB Specifically, we get these constants after some algebra from

$$
\begin{aligned}
\mathcal{N}_{S}^{-2} & =\frac{\Omega_{\Lambda}^{2}}{2 c_{t}^{2}}\left[\frac{c_{t}^{2}}{c_{l}^{2}} \frac{4 q^{2} L}{\cos ^{2}\left(k_{l} L / 2\right)}\left(1+\frac{\sin \left(k_{l} L\right)}{k_{l} L}\right)+\frac{\left(q^{2}-k_{t}^{2}\right)^{2} L}{k_{t}^{2} \cos ^{2}\left(k_{t} L / 2\right)}\left(1-\frac{\sin \left(k_{t} L\right)}{k_{t} L}\right)-8\left(q^{2}-k_{t}^{2}\right) \frac{\tan \left(k_{t} L / 2\right)}{k_{t}}\right], \\
\mathcal{N}_{A}^{-2} & =\frac{\Omega_{\Lambda}^{2}}{2 c_{t}^{2}}\left[\frac{c_{t}^{2}}{c_{l}^{2}} \frac{4 q^{2} L}{\sin ^{2}\left(k_{l} L / 2\right)}\left(1-\frac{\sin \left(k_{l} L\right)}{k_{l} L}\right)+\frac{\left(q^{2}-k_{t}^{2}\right)^{2} L}{k_{t}^{2} \sin ^{2}\left(k_{t} L / 2\right)}\left(1+\frac{\sin \left(k_{t} L\right)}{k_{t} L}\right)+8\left(q^{2}-k_{t}^{2}\right) \frac{\cot \left(k_{t} L / 2\right)}{k_{t}}\right] .
\end{aligned}
$$

\section{Appendix B: Linearized Boltzmann equation}

The linearized Boltzmann equation has been derived for closely related problems before, $\stackrel{30,35,36,44}{a}$ and we here follow those works and briefly sketch the derivation of Eq. (28). In the quasiclassical approximation, the quasiparticle distribution function $f(\mathbf{r}, \mathbf{k}, t)$ (for simplicity, we here omit the $v$ and $s$ indices) obeys the well-known Boltzmann equation. In the absence of a force $\mathbf{F}=-e \mathbf{E}$ due to the external electric field, $f$ reduces to a Fermi function, $f=n_{F}\left(\epsilon_{\mathbf{k}}\right)$, and the collision integral vanishes. In the presence of the force, $f$ is expanded in terms of Legendre polynomials $P_{n}(\cos \alpha)$, where $\alpha$ is the angle between $\mathbf{k}$ and $\mathbf{F}$. Keeping only terms linear in $\mathbf{F}$, we have $f(\mathbf{k})=n_{F}\left(\epsilon_{\mathbf{k}}\right)+\cos (\alpha) f_{1}\left(\epsilon_{\mathbf{k}}\right)$. Using detailed balance, for given transition matrix elements $W_{\mathbf{k} \rightarrow \mathbf{k}^{\prime}}$, we obtain the linearized Boltzmann equation (LBE),

$$
\begin{aligned}
& \mathbf{F} \cdot \mathbf{v}_{\mathbf{k}} \partial_{\epsilon} n_{F}\left(\epsilon_{\mathbf{k}}\right)=\sum_{\mathbf{k}^{\prime}} W_{\mathbf{k} \rightarrow \mathbf{k}^{\prime}} \\
\times & {\left[\frac{n_{F}\left(\epsilon_{\mathbf{k}}\right)}{n_{F}\left(\epsilon_{\mathbf{k}^{\prime}}\right)} \cos \left(\alpha^{\prime}\right) f_{1}\left(\epsilon_{\mathbf{k}^{\prime}}\right)-\frac{1-n_{F}\left(\epsilon_{\mathbf{k}^{\prime}}\right)}{1-n_{F}\left(\epsilon_{\mathbf{k}}\right)} \cos (\alpha) f_{1}\left(\epsilon_{\mathbf{k}}\right)\right] . }
\end{aligned}
$$

Using the Ansatz $f_{1}\left(\epsilon_{\mathbf{k}}\right)=-\tau\left(\epsilon_{\mathbf{k}}\right) v_{\mathbf{k}} F \partial_{\epsilon} n_{F}\left(\epsilon_{\mathbf{k}}\right)$, after some algebra the LBE leads to the linear integral equa- tion

$$
\frac{1}{\tau\left(\epsilon_{\mathbf{k}}\right)}=\sum_{\mathbf{k}^{\prime}} W_{\mathbf{k} \rightarrow \mathbf{k}^{\prime}}\left[1-\frac{v_{\mathbf{k}^{\prime}}}{v_{\mathbf{k}}} \frac{\tau\left(\epsilon_{\mathbf{k}^{\prime}}\right)}{\tau\left(\epsilon_{\mathbf{k}}\right)} \cos (\vartheta)\right] \frac{1-n_{F}\left(\epsilon_{\mathbf{k}^{\prime}}\right)}{1-n_{F}\left(\epsilon_{\mathbf{k}}\right)},
$$

where $\vartheta$ is the angle between $\mathbf{k}$ and $\mathbf{k}^{\prime}$. The solution for $\tau\left(\epsilon_{\mathbf{k}}\right)$ determines the electron momentum relaxation time. When the scattering of quasiparticles from long-wavelength acoustic phonons is quasielastic, $\Omega_{q, n} \ll|\mu|$, we can set $\tau\left(\epsilon_{\mathbf{k}^{\prime}}\right)=\tau\left(\epsilon_{\mathbf{k}}\right)$ for the right-hand side of the above integral equation; this is equivalent to the "test particle approximation." 30 The current density, $\mathbf{j}=-(e / \mathcal{A}) \sum_{\mathbf{k}} \mathbf{v}_{\mathbf{k}} f(\mathbf{k})$, points parallel to the electric field direction and has the magnitude

$$
j=\frac{e^{2} E}{\mathcal{A}} \sum_{\mathbf{k}} v_{\mathbf{k}}^{2} \cos ^{2}(\alpha) \tau\left(\epsilon_{\mathbf{k}}\right)\left[-\partial_{\epsilon} n_{F}\left(\epsilon_{\mathbf{k}}\right)\right] .
$$

Using $v_{\mathbf{k}}=v_{k}$ and performing the angular integration, we arrive at the phonon contribution to the resistivity quoted in Eqs. (28) and (29).

\section{Appendix C: Transport Eliashberg function}

We here give the analytical result for the full transport Eliashberg function $\mathcal{F}$ defined in Eq. (32). Some 


$$
\begin{aligned}
\mathcal{F}_{k, n, s}^{(\nu)}(q) & =\frac{2\left|A_{k, q, n, s}^{(\nu)}\right|}{\pi\left(A_{2} W\right)^{2}} \frac{\Theta\left(Q_{k, q, n, s}^{(\nu)}+k-q\right) \Theta\left(q-\left|Q_{k, q, n, s}^{(\nu)}-k\right|\right)}{\left[\left(q^{2}-\left(Q_{k, q, n, s}^{(\nu)}-k\right)^{2}\right)\left(\left(Q_{k, q, n, s}^{(\nu)}+k\right)^{2}-q^{2}\right)\right]^{1 / 2}} \Theta\left(\left|A_{k, q, n, s}^{(\nu)}\right|-\Delta / 2\right) \\
& \times\left.\sum_{s^{\prime}} \Theta\left(s^{\prime} A_{k, q, n, s}^{(\nu)}\right)\left[1-\left(1-\frac{\nu \Omega_{q, n}}{A_{k, q, n, s}^{(\nu)}}\right) \frac{\left(Q_{k, q, n, s}^{(\nu)}\right)^{2}+k^{2}-q^{2}}{2 k^{2}}\right]\left|M_{\mathbf{k}, \mathbf{q}, n}^{s^{\prime}, s}\right|^{2}\right|_{\theta_{0}},
\end{aligned}
$$

where we use the notations

$$
\begin{aligned}
A_{k, q, n, s}^{(\nu)} & =s \sqrt{(\Delta / 2)^{2}+\left(A_{2} W k\right)^{2}}+\nu \Omega_{q, n}, \\
Q_{k, q, n, s}^{(\nu)} & =\frac{\sqrt{\left(A_{k, q, n, s}^{(\nu)}\right)^{2}-(\Delta / 2)^{2}}}{A_{2} W},
\end{aligned}
$$

and the polar angle $\theta=\theta_{0} \in[0, \pi]$ follows from

$$
\sqrt{k^{2}+q^{2}+2 k q \cos \theta_{0}}=Q_{k, q, n, s}^{(\nu)},
$$

fixing the polar angle between $\mathbf{k}$ and $\mathbf{q}$ in the matrix element $M$.
1 M.Z. Hasan and C.L. Kane, Rev. Mod. Phys. 82, 3045 (2010).

2 X.L. Qi and S.C. Zhang, Rev. Mod. Phys. 83, 1057 (2011).

${ }^{3}$ L. Fu and C.L. Kane, Phys. Rev. B 76, 045302 (2007).

4 X.L. Qi, T.L. Hughes, and S.C. Zhang, Phys. Rev. B 78, 195424 (2008).

${ }^{5}$ D. Hsieh, Y. Xia, D. Qian, L. Wray, J.H. Dil, F. Meier, J. Osterwalder, L. Patthey, J.G. Checkelsky, N.P. Ong, A.V. Fedorov, H. Lin, A. Bansil, D. Grauer, Y.S. Hor, R.J. Cava, and M. Z. Hasan, Nature 460, 1101 (2009); Y.L. Chen, J.H. Chu, J.G. Analytis, Z.K. Liu, K. Igarashi, H.-H. Kuo, X.L. Qi, S.K. Mo, R.G. Moore, D.H. Lu, M. Hashimoto, T. Sasagawa, S.C. Zhang, I.R. Fisher, Z. Hussain, and Z.X. Shen, Science 329, 659 (2010); L.A. Wray, S.-Y. Xu, Y. Xia, Y.S. Hor, D. Qian, A.V. Fedorov, H. Lin, A. Bansil, R.J. Cava, and M.Z. Hasan, Nat. Phys. 6, 855 (2010); C. Jozwiak, Y.L. Chen, A.V. Fedorov, J.G. Analytis, C.R. Rotundu, A.K. Schmid, J.D. Denlinger, Y.-D. Chuang, D.H. Lee, I.R. Fisher, R.J. Birgeneau, Z.-X. Shen, Z. Hussain, and A. Lanzara, Phys. Rev. B 84, 165113 (2011).

${ }^{6}$ N.P. Butch, K. Kirshenbaum, P. Syers, A.B. Sushkov, G.S. Jenkins, H.D. Drew, and J. Paglione, Phys. Rev. B 81, 241301(R) (2010).

7 D.X. Qu, Y.S. Hor, J. Xiong, R.J. Cava, and N.P. Ong, Science 329, 821 (2010).

8 J.G. Analytis, R.D. McDonald, S.C. Riggs, J.-H. Chu, G.S. Boebinger, and I.R. Fisher, Nat. Phys. 6, 960 (2010).

9 B. Hellsing, A. Eiguren, and E.V. Chulkov, J. Phys. Cond. Matt. 14, 5959 (2002).

10 P.M. Echenique, R. Berndt, E.V. Chuklov, Th. Fauster, A. Goldmann, and U. Höfer, Surf. Sci. Rep. 52, 219 (2004).

11 S. Giraud and R. Egger, Phys. Rev. B 83, 245322 (2011).

12 C.X. Liu, X.L. Qi, H.J. Zhang, X. Dai, Z. Fang, and S.C. Zhang, Phys. Rev. B 82, 045122 (2010).

13 H. Zhang, C.X. Liu, X.L. Qi, X. Dai, Z. Fang, and S.C. Zhang, Nat. Phys. 5, 438 (2009).
14 W.Y. Shan, H.Z. Lu, and S.Q. Shen, New J. Phys. 12, 043048 (2010).

15 Y. Zhang, K. He, C.Z. Chang, C.L. Song, L.L. Wang, X. Chen, J.F. Jia, Z. Fang, X. Dai, W.Y. Shan, S.Q. Shen, Q. Niu, X.L. Qi, S.C. Zhang, X.C. Ma, and Q.K. Xue, Nat. Phys. 6, 584 (2010).

16 S. Cho, N.P. Butch, J. Paglione, and M.S. Fuhrer, Nano Lett. 11, 1925 (2011).

17 C.X. Liu, H.J. Zhang, B. Yan, X.L. Qi, T. Frauenheim, X. Dai, Z. Fang, and S.C. Zhang, Phys. Rev. B 81, 041307(R) (2010).

18 J. Linder, T. Yokoyama, and A. Sudbø, Phys. Rev. B 80, 205401 (2009).

19 K. Park, J.J. Heremans, V.W. Scarola, and D. Minic, Phys. Rev. Lett. 105, 186801 (2010).

20 O.V. Yazyev, J.E. Moore, and S.G. Louie, Phys. Rev. Lett. 105, 266806 (2010).

21 B. Seradjeh, J.E. Moore, and M. Franz, Phys. Rev. Lett. 103, 066402 (2009); G.Y. Cho and J.E. Moore, Phys. Rev. B 84, 165101 (2011).

22 H.K. Pal, V.I. Yudson, and D.L. Maslov, preprint arXiv:1108.2435.

23 S.R. Park, W.S. Jung, C. Kim, D.J. Song, C. Kim, S. Kimura, K.D. Lee, and N. Hur, Phys. Rev. B 81, 041405(R) (2010).

24 R.C. Hatch, M. Bianchi, D. Guan, S. Bao, J. Mi, B.B. Iversen, L. Nilsson, L. Hornekaer, and P. Hofmann, Phys. Rev. B 83, 241303(R) (2011).

25 Z.-H. Pan, A.V. Fedorov, D. Gardner, Y.S. Lee, S. Chu, and T. Valla, arXiv:1109.3638.

26 X. Zhu, L. Santos, R. Sankar, S. Chikara, C. Howard, F.C. Chou, C. Chamon, and M. El-Batanouny, Phys. Rev. Lett. 107, 186102 (2011).

27 L.D. Landau and E.M. Lifshitz, Elasticity Theory (Pergamon, New York, 1986).

28 J.O. Jenkins, J.A. Rayne, and R.W. Ure, Jr., Phys. Rev. 
B 5, 3171 (1972).

29 B.L. Huang and M. Kaviany, Phys. Rev. B 77, 125209 (2008).

30 N. Bannov, V. Aristov, V. Mitin, and M.A. Stroscio, Phys. Rev. B 51, 9930 (1995).

31 Y.M. Sirenko, K.W. Kim, and M.A. Stroscio, Phys. Rev. B 56, 15770 (1997).

32 P. Thalmeier, Phys. Rev. B 83, 125314 (2011).

${ }^{33}$ K.M.F. Shahil, M.Z. Hossain, D. Teweldebrhan, and A.A. Balandin, Appl. Phys. Lett. 96, 153103 (2010); J. Qi, X. Chen, W. Yu, P. Cadden-Zimansky, D. Smirnov, N.H. Tolk, I. Miotkowski, H. Cao, Y.P. Chen, Y. Wu, S. Qiao, and Z. Jiang, Appl. Phys. Lett. 97, 182102 (2010).

34 Ph. Hofmann, Prog. Surf. Sci. 81, 191 (2006).

35 E.H. Hwang and S. Das Sarma, Phys. Rev. B 77, 115449 (2008).

36 E. Mariani and F. von Oppen, Phys. Rev. B 82, 195403 (2010).

37 D.K. Efetov and P. Kim, Phys. Rev. Lett. 105, 256805 (2010).

38 G. Zhang, H. Qin, J. Teng, J. Guo, Q. Guo, X. Dai, Z. Fang, and K. Wu, Appl. Phys. Lett. 95, 053114 (2009).
39 G.E. Shoemaker, J.A. Rayne, and R.W. Ure, Phys. Rev. 185, 1046 (1969).

${ }^{40}$ W. Richter, H. Köhler, and C.R. Becker, phys. stat. sol. (b) 84, 619 (1977).

41 J.R. Wiese and L. Muldawer, J. Phys. Chem. Solids 15, 13 (1960).

${ }^{42}$ We mention that Eq. (8) in Ref. 11 contains a mistake: in the denominator of the expression for $C^{-1}$, one should replace $\kappa_{l}^{2} \kappa_{t} \rightarrow \kappa_{l} \kappa_{t}^{2}$. This implies $C \simeq 0.78$ instead of the quoted value $C \simeq 1.20$ in Ref. 11. Fortunately, this mistake has no consequences for the conclusions reached in Ref. 11 .

43 N.W. Ashcroft and N.D. Mermin, Solid State Physics (Saunders College, Philadelphia, 1976).

44 T. Kawamura and S. Das Sarma, Phys. Rev. B 45, 3612 (1992).

45 In principle, the self-energy is off-diagonal in the $s s^{\prime}$ indices, but for the rate $\Gamma$, only the diagonal element with $s=s^{\prime}$ is needed. We also use the fact that $\Sigma$ then is $v$ independent.

46 Y. Jiang, Y. Wang, M. Chen, Z. Li, C. Song, K. He, L. Wang, X. Chen, X. Ma, and Q.K. Xue, preprint arXiv:1111.1485. 